\title{
Translation of the synaptonemal complex component Sycp3 is enhanced in vivo by the germ cell specific regulator Dazl
}

\author{
NICOLA REYNOLDS, ${ }^{1}$ BRIAN COLLIER, ${ }^{1,2}$ VICTORIA BINGHAM, ${ }^{1}$ NICOLA K. GRAY, ${ }^{1,2}$ \\ and HOWARD J. COOKE ${ }^{1}$ \\ ${ }^{1}$ Medical Research Council Human Genetics Unit, Western General Hospital, Crewe Road, Edinburgh, EH4 2XU, United Kingdom \\ ${ }^{2}$ School of Clinical Sciences and Community Health, 47 Little France Crescent, EH16 4TJ, United Kingdom
}

\begin{abstract}
DAZ-related genes are essential for gametogenesis in diverse metazoa: in human males, a loss of DAZ genes is associated with infertility. These genes, expressed only in germ cells, regulate the translation of a yet undefined set of specific transcripts, and loss of function results in numerous defects throughout the mitotic and meiotic process of germ cell development. In a mouse model, absence of the autosomal Dazl gene results in a final block at zygotene of meiotic prophase. Sycp3 is also essential for meiosis, specifically for the formation of the synaptonemal complex lateral element with a mouse knockout model displaying a block in meiotic prophase similar to the Dazl knock out. Sycp3 was identified as a potential target for translational regulation by Dazl in male mouse germ cells. This was confirmed by both RNA binding and translation assays. In the Dazl knockout mouse model, Sycp3 protein levels were decreased, indicating that Dazl is required for efficient translation of the Sycp3 mRNA in vivo. Taken together these data support Sycp3 as a biologically relevant target of Dazl-mediated translation in mammals. This suggests that azoospermia associated with a decrease in $D A Z$ gene function in humans may in part be a consequence of failure at synapsis caused by reduced levels of SYCP3 protein.
\end{abstract}

Keywords: translation; gametogenesis; spermatogenesis; RNA binding proteins; infertility

\section{INTRODUCTION}

The very highly ordered process of gametogenesis is essential for the faithful transfer of genetic information from one generation to the next. In male mice, this entails mitotic proliferation of germ cells to establish and maintain a population of germline stem cells, followed by meiosis, which results in a reduction from diploid to haploid chromosome complement. Finally, cells undergo a differentiation process known as spermiogenesis to generate mature, motile sperm.

The control of developmental processes by posttranscriptional regulation, in the form of changes in mRNA stability, localization, or translation, is well documented. Germ cell development relies heavily on such post-transcriptional regulation, for example, in the regulation of maternal

Reprint requests to: Howard J. Cooke, Medical Research Council Human Genetics Unit, Western General Hospital, Crewe Road, Edinburgh, EH4 2XU, United Kingdom; e-mail: Howard.Cooke@hgu.mrc.ac.uk; fax: +44-131-467-8456.

Article published online ahead of print. Article and publication date are at http://www.rnajournal.org/cgi/doi/10.1261/rna.465507.
mRNAs during oocyte maturation and for translation of stored transcripts during spermiogenesis, a time of transcriptional silencing. This regulation utilizes a complex network of interactions between RNA binding proteins and regulatory sequences within the mRNA, usually located in the UTRs (for review, see Braun 1998; Gray and Wickens 1998; Kuersten and Goodwin 2003; de Moor et al. 2005; Ehrmann and Elliott 2005; Piccioni et al. 2005).

The deleted in azoospermia (DAZ) family of germ cell specific translational regulators is required for mitotic and meiotic progression in metazoans (Reijo et al. 1995; Eberhart et al. 1996; Ruggiu et al. 1997; Houston and King, 2000; Karashima et al. 2000) and consists of Dazl and Boule in vertebrates, with additional Y-chromosomal family members, $D A Z$ is present in humans and Old World primates. The $D A Z$ family genes encode RNA binding proteins that contain a conserved RNA recognition motif (RRM) and at least one copy of a DAZ motif that has been implicated in multiple protein-protein interactions (Tsui et al. 2000a; Dai et al. 2001; Moore et al. 2003, 2004). DAZ family proteins bind to U-rich sequences in specific 
transcripts and are required for their efficient translation in vivo (Houston et al. 1998; Tsui et al. 2000b; Venables et al. 2001; Jiao et al. 2002; Maegawa et al. 2002; Collier et al. 2005; Reynolds et al. 2005; Otori et al. 2006). They have been shown to act at the level of translation initiation by directly recruiting a translation initiation factor, polyA binding protein (PABP), to the mRNA through interaction with the 3' UTR of the message (Collier et al. 2005).

In addition to this interaction with PABP, DAZ family proteins also interact with a number of other proteins, including DAZAP1, DAZAP2, PUM2, hQK3, DZIP1, DZIP2, DZIP3, and Dynein light chain, many of which are RNA binding proteins as well as being capable of forming hetero- and homodimers with other DAZ family members (Ruggiu and Cooke 2000; Tsui et al. 2000a; Xu et al. 2001; Moore et al. 2003, 2004; Urano et al. 2005; Lee et al. 2006). The functional significance of many of these interactions remains to be clarified in vivo, although it seems probable that interactions with different proteins will have differing effects on post-transcription regulation of mRNAs, e.g., an interaction with PABP promotes translation, interaction with Pum2 and DAZAP1 (Moore et al. 2003; Urano et al. 2005; Morton et al. 2006) may be involved in translational repression, and interactions with dynein light chain may be required for specific localization of messages (Tsui et al. 2000a; Lee et al. 2006). It is possible that as specific translational requirements change throughout germ cell development, so, too, do the components of the complexes containing Dazl.

Several studies have been carried out to identify the RNA targets of Dazl proteins, although few have been able to address the biological significance of those interactions. It is clear, however, that there is a high level of binding specificity to particular RNA sequences (Venables et al. 2001; Jiao et al. 2002; Maegawa et al. 2002; Fox et al. 2005; Reynolds et al. 2005).

A correlation between loss of the Y-chromosomal $D A Z$ genes and infertility in humans has been well established (Reijo et al. 1995). Associations between loss of the other family members and human fertility levels in both males and females have also been suggested (Teng et al. 2002; Luetjens et al. 2004; Tung et al. 2006a,b). Male mice homozygous null for Dazl show a range of germ cell specific phenotypes, the precise nature of which are dependent on mouse strain background. This includes the loss of premeiotic germ cells before birth, a failure in the transition between $A_{\text {aligned }}$ and $A_{1}$ spermatogonia, and a final block in meiosis at zygotene of meiotic prophase I (Ruggiu et al. 1997; Schrans-Stassen et al. 2001; Saunders et al. 2003; Lin and Page 2005).

A block in meiotic progression at zygotene is reminiscent of the $S y c p 3$ null phenotype in male mice (Yuan et al. 2000). Synapsis of homologous chromosomes during zygotene and pachytene of meiotic prophase is essential for the efficient pairing, condensation, and recombination that must occur for meiosis to progress. This is reliant on the formation of an extensive protein complex, the synaptonemal complex, which holds homologous chromosomes together throughout prophase I. Synaptonemal complex formation begins with the formation of a lateral/axial element containing, among others, the proteins SYCP2 and SYCP3 during leptotene and is followed by the formation of the central element containing SYCE1, SYCE2/CESC1, and TEX12 and the transverse filaments containing SYCP1 during zygotene and pachytene (Meuwissen et al. 1992; Dobson et al. 1994; Lammers et al. 1994; Offenberg et al. 1998; Costa et al. 2005; Hamer et al. 2006).

As for the $D A Z$ family genes, loss of function of synaptonemal complex components in humans has been associated with infertility (Miyamoto et al. 2003; Judis et al. 2004; Sun et al. 2005; Topping et al. 2006). We recently identified the Sycp3 transcript as a potential target for translational regulation by mouse Dazl. Not only was the Sycp3 transcript found to be associated in vivo with Dazl protein in UV cross-linking immunoprecipitation experiments, but the 3' UTR of this mRNA also contains a conserved consensus sequence for Dazl binding (Reynolds et al. 2005). Here, we show that Dazl protein interacts in vitro with the 3' UTR of Sycp3 and stimulates its translation in vivo, in a manner that is dependent on the presence of this conserved Dazl binding sequence. Furthermore, in Dazl null animals, protein levels of Sycp3 were much reduced. This demonstrates that Dazl is required for the efficient translation of Sycp3 in vivo. We suggest that specific features of the Dazl null phenotype may be explained by a failure to achieve adequate Sycp3 protein levels, an observation that may be extended to the azoospermic phenotype in men lacking $D A Z$ gene function. Sycp3 is therefore only the second mammalian target for Dazl-mediated translation shown to be biologically relevant in vivo.

\section{RESULTS}

It has been shown previously that endogenous Dazl protein associates with Sycp3 mRNA in mouse testis (Reynolds et al. 2005). The $3^{\prime}$ UTR of Sycp3 contains a consensus sequence for Dazl binding $\left(\mathrm{U}_{2-10}[\mathrm{G} / \mathrm{C}] \mathrm{U}_{2-10}\right)$ that is conserved in the 3' UTR of rat Sycp3 (Fig. 1A; Reynolds et al. 2005). Given the similarity in phenotype of animals null for either Dazl or Sycp3, we investigated this interaction in vitro.

Binding was assessed by mixing biotinylated RNA corresponding to the 3' UTR of Sycp3 with a variety of recombinant Dazl proteins in rodent testis homogenate. Testis homogenate was used to mimic endogenous competition conditions for the interaction assay. RNA was isolated using Streptavidin-linked magnetic beads, and the presence or absence of recombinant Dazl protein was determined by Western blotting. In this assay, the wild-type Sycp3 3' UTR sequence bound equally well to both full-length T7-tagged 
A

1 ugagucuuugaagaaagaacuugaaccuauguaauaugauacaauua aaacauuagcuaagaggcaugccuuuaguaaauuauaguuuaacuauaaua uccgaaaucauuagcuuauuuaaguggaagauuuuuguuccuguuaaccuaa aaccuuauaaaccuaaacuauguuaaguaccagcuauuccaauguaucaaac uuucaggguuuuuuguuuguuugUUUUAAUaguuguucucuccacgauugug ucaauaaagaugauuuaaauuu 277
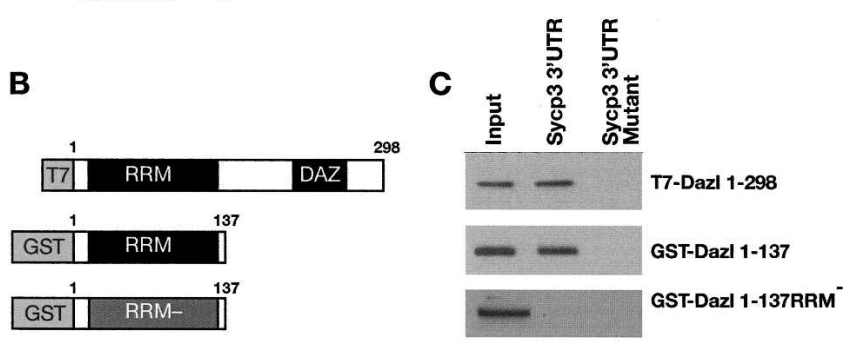

D

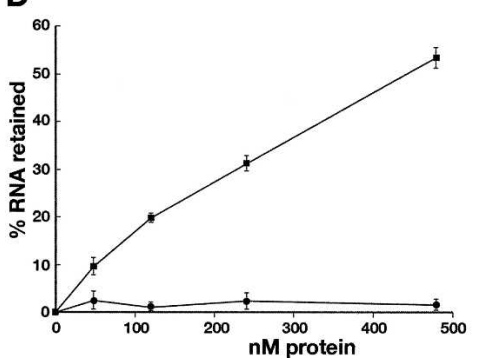

FIGURE 1. (A) The 3' UTR of Sycp3 contains a consensus Dazl binding sequence (bold type and underlined) which is conserved in rodents. The CPEB binding site (Tay and Richter 2001) is capitalized, and the hexanucelotide is underlined with a discontinuous line. (B) Schematic of recombinant Dazl proteins used in binding assays. RRM - contains point mutations in the RRM which prevent binding to RNA. (C) Western blots showing association of recombinant fulllength and truncated (1-137) Dazl with biotinylated Sycp3 RNA but not with RNA in which the Dazl binding sequence had been mutated. $(D)$ Filter binding assays using recombinant GST-tagged truncated Dazl (1-137) and either sense (solid squares) or anti-sense (solid circles) Sycp3 3' UTR.

Dazl or a GST-tagged, truncated form of Dazl (residues 1-137) containing the RNA binding domain (RNA recognition motif/RRM) but not the DAZ motif. No binding was detected to truncated protein which carried point mutations in the RRM (Fig. 1B,C) or when antisense RNA was used as a control (data not shown). This shows that the RNA binding specificity of Dazl relies solely on the integrity of the RRM and does not require the DAZ motif. No association between any of the Dazl proteins was detected with Sycp3 3' UTR RNA in which the consensus Dazl binding site had been mutated from $\mathrm{U}_{6} \mathrm{GU}_{3} \mathrm{GU}_{3} \mathrm{GU}_{4}$ to $\mathrm{C}_{6} \mathrm{UC}_{3} \mathrm{UC}_{3} \mathrm{UC}_{4}$ (Fig. 1C), demonstrating that this motif is absolutely required for the interaction in vitro. Filter binding assays showed that the affinity of GST-Dazl ${ }_{1-137}$ for Sycp3 3' UTR was comparable to that of Mvh 3' UTR, the only previous in vivo characterized mammalian target (Fig. 1D; Reynolds et al. 2005). Importantly, these assays also established that binding was direct and independent of other proteins (Fig. 1D).

Dazl has been shown to stimulate translation at the level of initiation of translation when bound to the 3' UTR of mRNAs (Collier et al. 2005; Reynolds et al. 2005). A biologically relevant interaction might thus be expected to result in stimulation of translation (Collier et al. 2005; Reynolds et al. 2005). To test this we used a Xenopus oocyte based assay to establish the role of Dazl protein in translation. A fourfold increase in luciferase expression was seen when the wild-type $S y c p 33^{\prime}$ UTR was fused to the $3^{\prime}$ end of the luciferase reporter in the presence of Dazl protein, relative to an unrelated RNA binding protein, U1A. No increase in the expression of the internal control $\beta$-galactosidase mRNA or a luciferase mRNA containing an unrelated 3' UTR (data not shown; Fig. 2A) was observed, demonstrating specificity of the effect. Importantly, reporter mRNA levels remain unchanged over the course of the assay, showing that the increase in luciferase activity is a direct result of an increase in translation rather than changes in the stability of the RNA (Fig. 2B). To test whether the Dazl consensus sequence was required for this effect, the mutant Sycp3 UTR in Figure 1 was appended to the luciferase mRNA. No such increase in translation was seen when the Sycp3 3' UTR containing mutations in the Dazl binding site was used (Fig. 2C), establishing a direct link between Dazl binding and translational stimulation.

In order to definitively show that this interaction is of biological significance, Sycp3 protein levels in Dazl null mice were measured. In male mice, the first wave of spermatogenesis proceeds synchronously, starting at around day 7 after birth and taking $\sim 35 \mathrm{~d}$ to complete (Goetz et al. 1984). Entry into and progression through meiosis can therefore be studied by examining testes from animals in the first weeks after birth. However, in an inbred C57Bl/6 strain background, Dazl null mice lose germ cells embryonically, at E13.5, and few or no germ cells can be detected after birth (Fig. 3A; Lin and Page 2005). This is before significant levels of Sycp3 protein are present in wild-type animals.

Dazl null male mice with outbred genetic backgrounds are born with morphologically normal germ cells. Progression through meiosis has been observed up until zygotene of meiotic prophase I (Ruggiu et al. 1997; Saunders et al. 2003). In order to observe early meiotic processes in Dazl null animals, we crossed C57Bl/6 Dazl ${ }^{+/-}$males with CD1 Dazl ${ }^{+/+}$females and intercrossed the resultant progeny to produce $\mathrm{Dazl}^{-1-}$ animals with a reproducible, outbred genetic background.

The germ cell complement was assessed by staining with an antibody directed against the germ cell marker, GCNA (Enders and May 1994). In the outbred background, germ cells persisted after birth in Dazl null male mice, although there was considerable variation between individual animals with respect to the number of tubules containing 
A

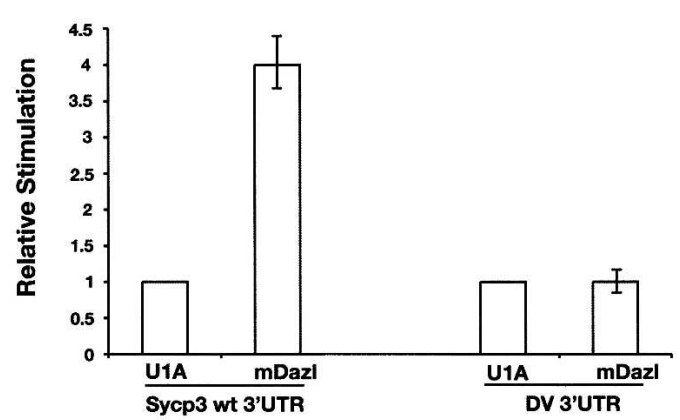

B

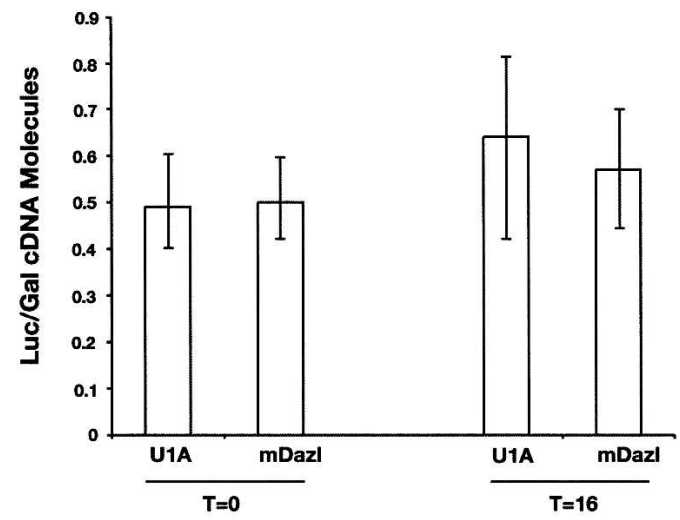

C

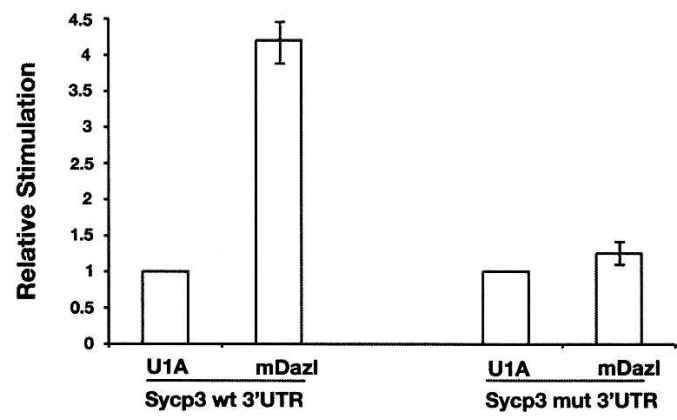

FIGURE 2. (A) Mouse Dazl stimulates translation of a luciferase reporter mRNA relative to an unrelated RNA binding protein, U1A, in the presence of Sycp3 3' UTR but not the unrelated 3' UTR of Dengue virus (DV). Neither of the luciferase reporter mRNAs is adenylated. $(B)$ Mouse Dazl does not affect mRNA stability. Relative numbers of molecules of luciferase normalized to $\beta$-galactosidase cDNA are shown in the presence or absence of mouse Dazl at time points zero $(T=0)$ and $16 \mathrm{~h}(T=16)$ postinjection and after RNA extraction and reverse transcription. (C) Consensus Dazl binding sites are required for Sycp3 translational regulation. Dazl stimulates the translation of luciferase mRNA with the Sycp3 wild-type but not mutant $3^{\prime}$ UTR. For $(A)$ and $(C)$ luciferase values are normalized to a co-injected $\beta$-galactosidase mRNA and normalized luciferase activity is plotted, with a value of U1A set to one. Error bars indicate the standard error of the mean.

germ cells. This level of variability reflects the heterogenous genetic background (Fig. 3B).

In all $\mathrm{Dazl}^{+/+}$animals examined at each age, $100 \%$ of the tubules contained a full complement of GCNA-positive cells (data not shown). In Dazl ${ }^{-1-}$ mice, GCNA positive cells were detected as late as 3 weeks after birth although normal meiotic progression was not apparent after $7 \mathrm{~d}$ post-partum (pp), as assessed by the morphology and position of these GCNA positive cells within the testis tubules (Fig. 3B,C). This is consistent with previous reports of a block at the leptotene/zygotene transition of meiotic prophase I in the absence of Dazl (Saunders et al. 2003).

Even in this outbred background there were generally fewer germ cells present in Dazl null animals when compared to wild type. Because of the large variation in germ

A

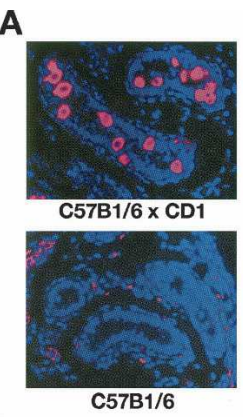

B

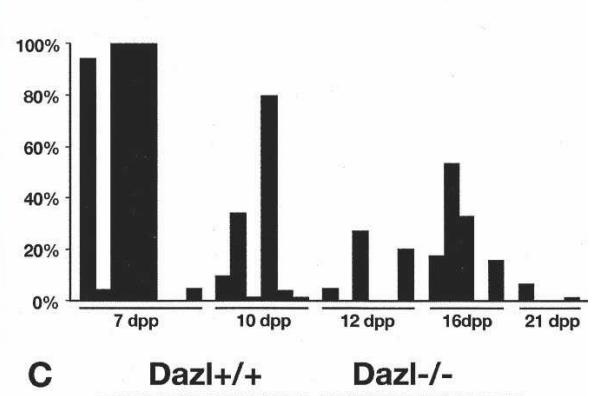

$7 \mathrm{dpp}$

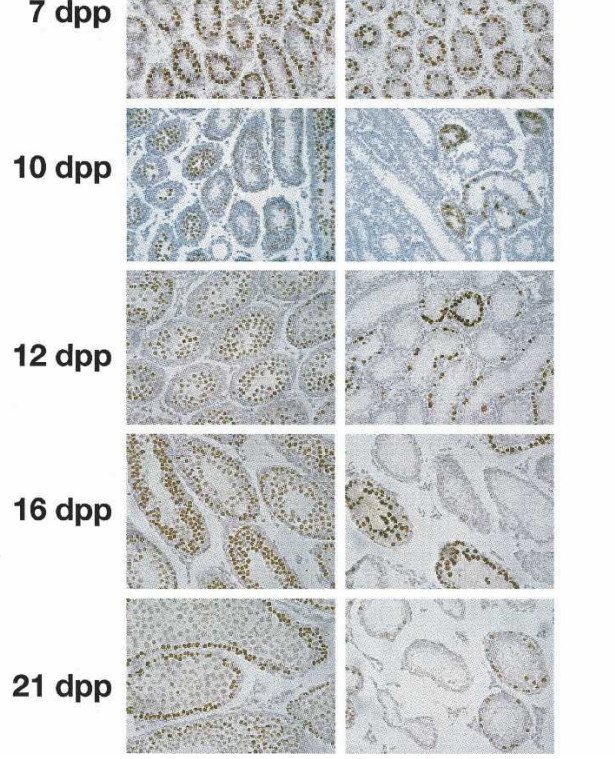

FIGURE 3. (A) Germ cells survive beyond birth in male Dazl null animals with an outbred genetic background $(\mathrm{C} 57 \mathrm{Bl} / 6 \times \mathrm{CD} 1)$ but not inbred $\mathrm{C} 57 \mathrm{Bl} / 6$. Testes were taken at $1 \mathrm{~d}$ post partum and stained with anti-Mvh antibody to detect germ cells (red) and counterstained with DAPI (blue). (B) Germ cell survival in outbred Dazl null male mice. Testes were taken at varying times after birth, fixed, and stained with anti-GCNA antibody. The percentage of tubules present containing GCNA-positive cells (brown staining) was determined for individual animals. $(C)$ Representative images showing GCNA-positive cells in $\mathrm{Dazl}^{+/+}$and ${ }^{-/-}$animals with $\mathrm{C} 57 \mathrm{Bl} / 6 \times \mathrm{CD} 1$ genetic background. Meiotic progression appears normal up until $7 \mathrm{~d}$ pp for those germ cells that remain in the null animals. From $10 \mathrm{~d} p \mathrm{pp}$ onward, GCNA-positive cells remain at edges of tubules and/or appear apoptotic, indicating that progression beyond this stage does not occur at any appreciable rate. 
cell numbers, measurements were made on a cell-by-cell basis for individual animals in order to compare absolute protein levels between genotypes using quantitative microscopy with identical staining, exposure, and capture conditions.

We were able to select animals at $7 \mathrm{~d}$ pp with a normal or close to normal germ cell complement for quantitative immunofluorescence. The amount of Sycp3 protein present in each cell was measured relative to phosphorylated $\mathrm{H} 2 \mathrm{AX}$ $(\gamma \mathrm{H} 2 \mathrm{AX})$. The localization pattern of $\gamma \mathrm{H} 2 \mathrm{AX}$ changes as cells progress through meiotic prophase, enabling only germ cells at equivalent stages of meiosis to be included in the analysis (Fig. 4). This time point was chosen because morphologically normal germ cells were present in most of the Dazl null animals tested. We therefore concluded that those germ cells present in the knockout animals were equivalent to those of wild-type animals and therefore

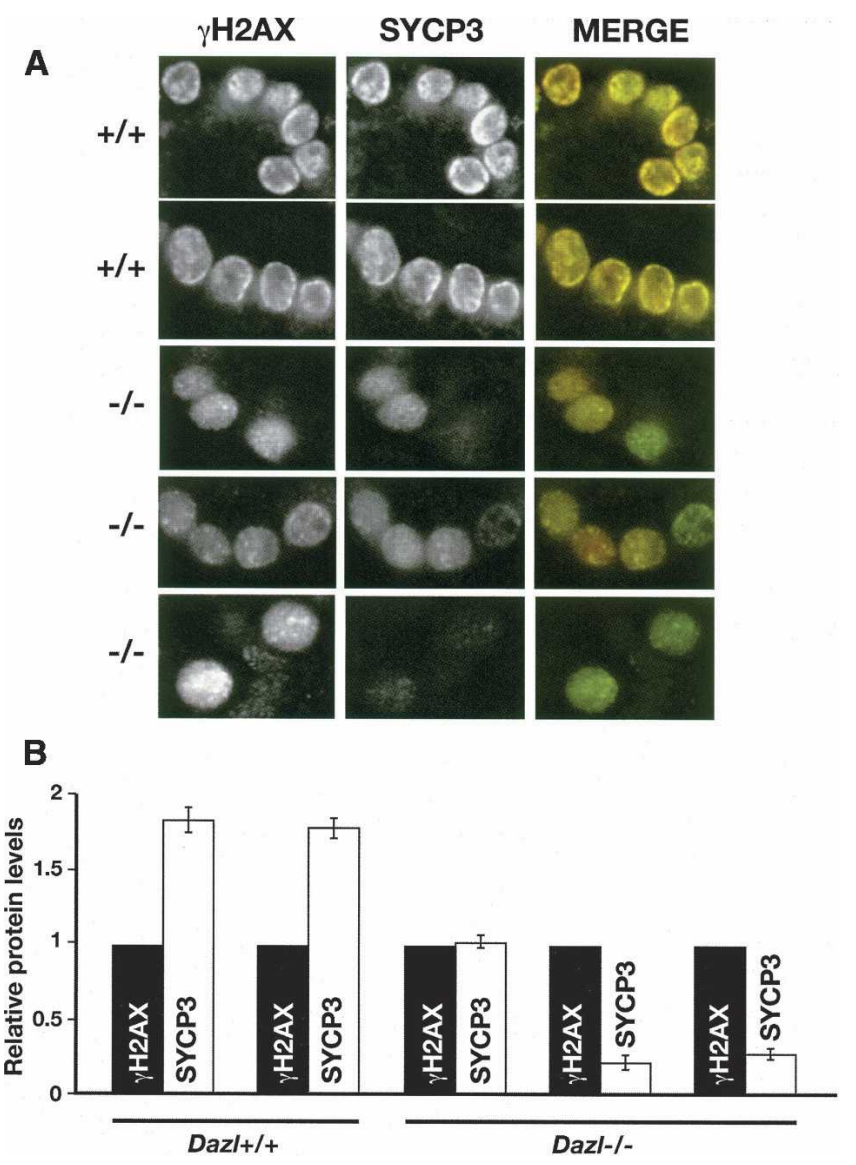

FIGURE 4. Quantitative immunofluorescence to measure relative levels of Sycp3 protein in germ cells from $\mathrm{Dazl}^{+/+}$and ${ }^{-/-}$testes $7 \mathrm{~d}$ pp. (A) Representative images taken under identical exposure conditions illustrating heterogenous loss of Sycp3 protein levels in germ cells from Dazl null animals $\left(^{-I-}\right)$. In the merged image, Sycp3 is shown in red and $\gamma \mathrm{H} 2 \mathrm{AX}$ in green. (B) Pixel intensity was measured at the level of individual cells (40 cells per animal) for both Sycp3 staining and $\gamma \mathrm{H} 2 \mathrm{AX}$, carried out under identical staining and exposure conditions. Sycp3 levels are shown normalized to $\gamma \mathrm{H} 2 \mathrm{AX}$ levels for individual animals. Error bars indicate standard error of the mean. could be compared directly. It is also around the time when a general cessation of normal meiotic progression was observed, and we postulated that more marked changes in protein levels might be seen as a consequence.

Rather than universal lack of Sycp3 protein in Dazl null animals, levels varied between cells. Some cells had apparently wild-type levels of Sycp3 protein, while other, neighboring cells contained barely detectable amounts (Fig. 4). In samples from wild-type animals, no cells were detected that were positive for $\gamma \mathrm{H} 2 \mathrm{AX}$ but negative for Sycp3. The variable protein levels in Dazl null animals mimic the situation for Mvh protein in testes of Dazl null mice, the only other mammalian Dazl target that has so far been characterized in vivo (Reynolds et al. 2005).

For three individual Dazl null animals examined, average levels of Sycp3 protein in germ cells for individual animals ranged from $55 \%$ of wild-type levels to $11 \%$, relative to $\gamma \mathrm{H} 2 \mathrm{AX}$ (Fig. 4B), consistent with DAZL enhancing rather than being an absolute requirement for the translation of these mRNAs. Levels of Sycp3 protein in wild-type animals remained comparatively constant from animal to animal. While we do not exclude the possibility that $\gamma \mathrm{H} 2 \mathrm{AX}$ itself is translationally regulated by Dazl, these data indirectly suggest that it is not, or that if it is, regulation is not important until later in meiosis.

The same trend was seen when measurements were made both at $10 \mathrm{~d}$ pp and when using GCNA as a reference for germ cell protein levels (data not shown). These data confirm that in the absence of Dazl protein in vivo levels of Sycp3 protein are reduced and support the hypothesis that Dazl mediates Sycp3 translation.

\section{DISCUSSION}

This study provides compelling evidence that Sycp3 is an in vivo target of Dazl-mediated translation in the male germline. Dazl null mice exhibit multiple meiotic phenotypes. A failure in synaptonemal complex formation caused by inadequate levels of Sycp3 protein is likely to contribute to one of these specific defects. In the absence of Sycp3 protein, female mice exhibit defective chromosome segregation, resulting in aneuploidy (Yuan et al. 2002), while a male specific block in meiotic prophase at zygotene is seen (Yuan et al. 2000). One of the defects in Dazl null mice is an equivalent arrest at zygotene evident in mice of both sexes. This suggests that there are other mRNAs that require Dazl for their efficient translation, particularly during female gametogenesis, which remain to be identified.

Although there are many well-characterized examples of regulated translation of maternal transcripts during oocyte maturation and early embryonic development and of stored transcripts in haploid spermatids, much less is known about the precise nature of Dazl-mediated translation in premeiotic and meiotic cells. However, recent work has suggested a role for the translation initiation 
factor, PABP (Collier et al. 2005) in Dazl-mediated regulation. In this model, Dazl directly recruits PABP to the $3^{\prime}$ end of mRNAs. This is reminiscent of the activation of mRNAs by cytoplasmic polyadenylation, a well-characterized mechanism of translational control that is of critical importance during gametogenesis and early embryogenesis (Gray and Wickens 1998). The parallels between poly(A) and Dazl-mediated recruitment of PABP raise the possibility that Dazl is likely to be of particular importance for mRNAs with short poly(A) tails that would not otherwise efficiently recruit PABP (Collier et al. 2005).

In this respect, it is interesting to note that Sycp3 mRNA is stored with a short poly(A) tail of only $\sim 20$ nucleotides and undergoes cytoplasmic polyadenylation (Tay and Richter, 2001), with variation in polyadenylation perhaps contributing to the observed variation in SYCP3 levels (Fig. 4). This raises the possibility that Sycp3 may require both cytoplasmic polyadenylation and Dazl-mediated recruitment of PABP for its full level of translational activation, an idea that is supported by the finding that adenylated reporter mRNAs bound by Dazl are translated to a higher level than those lacking a poly(A) tail (Collier et al. 2005). This idea is also in keeping with the lower level of Sycp3 translation in both CPEB and Dazl male knockout mice. However, the binding sites for Dazl and CPEB1 in the Sycp3 3' UTR overlap (Fig. 1A), making it questionable whether the two proteins would be able to bind to the 3' UTR simultaneously, although a physical interaction between DAZ-1 and CPB-3 has been reported in Caenorhabditis elegans (Hasegawa et al. 2006). By immunofluorescence, we were able to detect only a low level of colocalization between Dazl and CPEB1 proteins in the cytoplasm of mouse spermatocytes and have been unable to demonstrate a physical interaction between the two by coimmunoprecipitation (data not shown). Thus it is possible that Dazl and CPEB1 act sequentially to control Sycp3 translation in vivo. Moreover, it should be remembered that CPEB, like Pumilio, is also involved in translational repression and that the precise composition of Dazl complexes may determine whether the translation of a particular message is enhanced or repressed. Furthermore, it is possible that Dazlmediated regulation of some mRNAs may result in direct or indirect changes in RNA stability or even localization.

In the absence of Dazl in the mouse male germline, translation of target transcripts continues at a low level since proteins such as Mvh and Sycp3 are still produced, albeit at reduced levels when compared to wild type (this study; Reynolds et al. 2005). It seems that only upon failure to reach a threshold level of a particular protein, or when a number of proteins are present at insufficient levels, do cells arrest and/or die. This would account for the complex phenotype seen in Dazl null mice. Moreover, survival of Dazl null germ cells was enhanced in an outbred background, indicating that there are modifiers of the phenotype, perhaps in the form of other DAZ family members, other RNA binding proteins, or more general translational regulators. Identification of these would provide valuable insight into the mechanism of action of DAZ family proteins.

\section{MATERIALS AND METHODS}

\section{RNA protein interaction assays}

Assays were carried out as described previously (Reynolds et al. 2005). Bacterially produced, GST-tagged, truncated Dazl proteins (wild type and point mutated) have been described elsewhere (Reynolds et al. 2005), while T7-tagged, full-length Dazl was produced by transfecting the plasmid pCGDazl into $293 \mathrm{~T}$ cells according to Cazalla et al. (2005). The full-length Sycp3 3' UTR was cloned by PCR from adult mouse testis cDNA into pGEM-T Easy (Promega) to create pGEMTEasySycp3 3' UTR, which was sequenced fully. Mutations were introduced by PCR, products cloned into pGEM-T Easy, and inserts sequenced fully. Transcription from linearized plasmid was carried out using either T7 or SP6 RNA Polymerase-Plus (Ambion) as appropriate. Oligonucleotide sequences used were designed to the Sycp3 3' UTR sequence NM_011517 as follows:

\section{Forward: TGAGTCTTTGAAGAAAGAACTTGA and either WT-reverse: AAATTTAAATCATCTTTATTGACACA or mutant-reverse: AAATTTAAATCATCTTTATTGACACAATCGTG GAGAGGGAGGCTATTGGGGAGGGCGGGA.}

Proteins were detected with antibodies directed against either GST (Sigma) or T7 (Novagen). Filter binding assays were performed as described by Reynolds et al. (2005).

\section{Translation assays}

Translation assays using mRNAs microinjected into stage VI Xenopus oocytes were carried out as described using the plasmids pUC19LUC-Scp3wt and pUC19LUC-Scp3mut, which were created by inserting the 3' UTR of Sycp3 as a SalI-HindIII fragment into the vector pUC19LUC (Reynolds et al. 2005). pUC19LucDV(3' UTR) is described elsewhere (Reynolds et al. 2005).

\section{mRNA stability assays}

Total RNA was extracted from pools of oocytes immediately and $16 \mathrm{~h}$ following injection of the reporter mRNAs using Tri-Reagent (Sigma) as per the manufacturer's instructions. RNAs were reverse transcribed using an Omniscript RT kit (Qiagen) using reverse primers specific for either the luciferase (AGCGTTTTCCCGGTA TCCA) or $\beta$-galactosidase reporter (CTGATTTGTGTGGTCGGT TTGT).

Quantitative PCR was performed using an ABI Prism 7900HT Sequence Detection System (Applied Biosystems). The number of molecules of each reporter cDNA per sample was measured by incorporation of SYBR green into PCR products using SYBR GREEN PCR Master Mix (Applied Biosystems) and the following primers: Luc 5' (GGCGCGGTCGGTAAAGTT) and Luc 3' (AGCG TTTTCCCGGTATCCA) for luciferase, and Gal 5' (TCAGGATAT GTGGCGGATGA) and Gal 3' (CTGATTTGTGTGGTCGGTTTGT) 
for $\beta$-galactosidase. Data analysis was performed with the ABI Prism SDS2.1 software (Applied Biosystems), and standard curves generated from luciferase and $\beta$-galactosidase DNA standards were used to determine number of molecules in the test samples. All samples were analyzed in triplicate.

\section{Immunofluorescence and quantification of protein levels}

Antibodies used were rat anti-GCNA (a gift from G.C. Enders [Enders and May 1994]), rabbit anti-Sycp3 (a gift from C. Heyting [Lammers et al. 1994]), rabbit anti-Mvh (Abcam), and mouse anti-phosphohistone H2A.X, clone JBW301 (Upstate).

Imaging was carried out as described previously using constant exposure times for each image. Subsequent quantification was performed using IPLab Spectrum software (Scanalytics Corp.) (Reynolds et al. 2005).

\section{Animals and tissue collection}

Dazl $^{\text {Tm1hgu/Tmlhgu }}$ mice (Ruggiu et al. 1997) were housed under standard conditions and fed ad libitum. Generation and breeding of mice was covered by Home Office Project Licence 603067 and was approved by institutional ethics committees.

C57Bl/6 inbred mice used were from a line that had been backcrossed to pure $\mathrm{C} 57 \mathrm{Bl} / 6$ mice a minimum of eight times.

Outbred lines were established by mating C57Bl/6 Dazl ${ }^{+/-}$ male mice with CD1 females and intercrossing the resultant $\mathrm{Dazl}^{+/-}$animals to produce homozygous ${ }^{+/+}$and ${ }^{-/-}$animals.

Testes were recovered from mice at varying times after birth (with the day of birth designated $0.5 \mathrm{~d}$ post-partum) and fixed in $4 \%$ PFA. Tail tips were collected at the time of death for genotyping by PCR. Testes were fixed in 4\% PFA for 2-6 h depending on size before dehydration and mounting in paraffin wax. Sections $(0.6 \mu \mathrm{m})$ were cut and processed using standard methods for immunofluorescence or immunohistochemistry.

\section{ACKNOWLEDGMENTS}

We thank members of the Cooke laboratory, past and present, for useful discussions and critical reading of the manuscript. We also thank Mary Taggart and Rob Speed for technical assistance, Janice Young for help with animal husbandry, Susan Lynas and William Richardson for technical assistance with Xenopus, Paul Perry for help with microscopy, and Sandy Bruce for figure production. Professor G.C. Enders and Professor C. Heyting kindly provided antibodies. This work was funded by the U.K. Medical Research Council. B.C. and N.K.G. are funded by a Wellcome Trust Project grant and an MRC Senior Fellowship awarded to N.K.G.

Received January 17, 2007; accepted April 24, 2007.

\section{REFERENCES}

Braun, R.E. 1998. Post-transcriptional control of gene expression during spermatogenesis. Semin. Cell Dev. Biol. 9: 483-489.

Cazalla, D., Sanford, J.R., and Caceres, J.F. 2005. A rapid and efficient protocol to purify biologically active recombinant proteins from mammalian cells. Protein Expr. Purif. 42: 54-58.
Collier, B., Gorgoni, B., Loveridge, C., Cooke, H.J., and Gray, N.K. 2005. The DAZL family proteins are PABP-binding proteins that regulate translation in germ cells. EMBO J. 24: 2656-2666.

Costa, Y., Speed, R., Ollinger, R., Alsheimer, M., Semple, C.A., Gautier, P., Maratou, K., Novak, I., Hoog, C., Benavente, R., et al. 2005. Two novel proteins recruited by synaptonemal complex protein 1 (SYCP1) are at the centre of meiosis. J. Cell Sci. 118: 2755-2762.

Dai, T., Vera, Y., Salido, E.C., and Yen, P.H. 2001. Characterization of the mouse Dazapl gene encoding an RNA-binding protein that interacts with infertility factors DAZ and DAZL. BMC Genomics 2: 6.

de Moor, C.H., Meijer, H., and Lissenden, S. 2005. Mechanisms of translational control by the 3' UTR in development and differentiation. Semin. Cell Dev. Biol. 16: 49-58.

Dobson, M.J., Pearlman, R.E., Karaiskakis, A., Spyropoulos, B., and Moens, P.B. 1994. Synaptonemal complex proteins: occurrence, epitope mapping, and chromosome disjunction. J. Cell Sci. 107: 2749-2760.

Eberhart, C.G., Maines, J.Z., and Wasserman, S.A. 1996. Meiotic cell cycle requirement for a fly homologue of human deleted in azoospermia. Nature 381: 783-785.

Ehrmann, I. and Elliott, D.J. 2005. Post-transcriptional control in the male germ line. Reprod. Biomed. Online 10: 55-63.

Enders, G.C. and May, J.J. 1994. Developmentally regulated expression of a mouse germ cell nuclear antigen examined from embryonic day 11 to adult in male and female mice. Dev. Biol. 163: 331-340.

Fox, M., Urano, J., and Reijo Pera, R.A. 2005. Identification and characterization of RNA sequences to which human PUMILIO-2 (PUM2) and deleted in azoospermia-like (DAZL) bind. Genomics 85: 92-105.

Goetz, P., Chandley, A.C., and Speed, R.M. 1984. Morphological and temporal sequence of meiotic prophase development at puberty in the male mouse. J. Cell Sci. 65: 249-263.

Gray, N.K. and Wickens, M. 1998. Control of translation initiation in animals. Annu. Rev. Cell Dev. Biol. 14: 399-458.

Hamer, G., Gell, K., Kouznetsova, A., Novak, I., Benavente, R., and Hoog, C. 2006. Characterization of a novel meiosis-specific protein within the central element of the synaptonemal complex. J. Cell Sci. 119: 4025-4032.

Hasegawa, E., Karashima, T., Sumiyoshi, E., and Yamamoto, M. 2006. C. elegans CPB-3 interacts with DAZ-1 and functions in multiple steps of germline development. Dev. Biol. 295: 689-699.

Houston, D.W. and King, M.L. 2000. A critical role for Xdazl, a germ plasm-localized RNA, in the differentiation of primordial germ cells in Xenopus. Development 127: 447-456.

Houston, D.W., Zhang, J., Maines, J.Z., Wasserman, S.A., and King, M.L. 1998. A Xenopus DAZ-like gene encodes an RNA component of germ plasm and is a functional homologue of Drosophila boule. Development 125: 171-180.

Jiao, X., Trifillis, P., and Kiledjian, M. 2002. Identification of target messenger RNA substrates for the murine deleted in azoospermialike RNA-binding protein. Biol. Reprod. 66: 475-485.

Judis, L., Chan, E.R., Schwartz, S., Seftel, A., and Hassold, T. 2004. Meiosis I arrest and azoospermia in an infertile male explained by failure of formation of a component of the synaptonemal complex. Fertil. Steril. 81: 205-209.

Karashima, T., Sugimoto, A., and Yamamoto, M. 2000. Caenorhabditis elegans homologue of the human azoospermia factor DAZ is required for oogenesis but not for spermatogenesis. Development 127: 1069-1079.

Kuersten, S. and Goodwin, E.B. 2003. The power of the 3' UTR: translational control and development. Nat. Rev. Genet. 4: 626-637.

Lammers, J.H., Offenberg, H.H., van Aalderen, M., Vink, A.C., Dietrich, A.J., and Heyting, C. 1994. The gene encoding a major component of the lateral elements of synaptonemal complexes of the rat is related to X-linked lymphocyte-regulated genes. Mol. Cell. Biol. 14: 1137-1146. 
Lee, K.H., Lee, S., Kim, B., Chang, S., Kim, S.W., Paick, J.S., and Rhee, K. 2006. Dazl can bind to dynein motor complex and may play a role in transport of specific mRNAs. EMBO J. 25: 4263-4270.

Lin, Y. and Page, D.C. 2005. Dazl deficiency leads to embryonic arrest of germ cell development in XY C57BL/6 mice. Dev. Biol. 288: 309-316.

Luetjens, C.M., Xu, E.Y., Reijo Pera, R.A., Kamischke, A., Nieschlag, E., and Gromoll, J. 2004. Association of meiotic arrest with lack of BOULE protein expression in infertile men. J. Clin. Endocrinol. Metab. 89: 1926-1933.

Maegawa, S., Yamashita, M., Yasuda, K., and Inoue, K. 2002. Zebrafish DAZ-like protein controls translation via the sequence "GUUC." Genes Cells 7: 971-984.

Meuwissen, R.L., Offenberg, H.H., Dietrich, A.J., Riesewijk, A., van Iersel, M., and Heyting, C. 1992. A coiled-coil related protein specific for synapsed regions of meiotic prophase chromosomes. EMBO J. 11: 5091-5100.

Miyamoto, T., Hasuike, S., Yogev, L., Maduro, M.R., Ishikawa, M., Westphal, H., and Lamb, D.J. 2003. Azoospermia in patients heterozygous for a mutation in SYCP3. Lancet 362: 1714-1719.

Moore, F.L., Jaruzelska, J., Dorfman, D.M., and Reijo-Pera, R.A. 2004. Identification of a novel gene, DZIP (DAZ-interacting protein), that encodes a protein that interacts with DAZ (deleted in azoospermia) and is expressed in embryonic stem cells and germ cells. Genomics 83: 834-843.

Moore, F.L., Jaruzelska, J., Fox, M.S., Urano, J., Firpo, M.T., Turek, P.J., Dorfman, D.M., and Pera, R.A. 2003. Human Pumilio-2 is expressed in embryonic stem cells and germ cells and interacts with DAZ (deleted in azoospermia) and DAZ-like proteins. Proc. Natl. Acad. Sci. 100: 538-543.

Morton, S., Yang, H.T., Moleleki, N., Campbell, D.G., Cohen, P., and Rousseau, S. 2006. Phosphorylation of the ARE-binding protein DAZAP1 by ERK2 induces its dissociation from DAZ. Biochem. J. 399: 265-273.

Offenberg, H.H., Schalk, J.A., Meuwissen, R.L., van Aalderen, M., Kester, H.A., Dietrich, A.J., and Heyting, C. 1998. SCP2: a major protein component of the axial elements of synaptonemal complexes of the rat. Nucleic Acids Res. 26: 2572-2579.

Otori, M., Karashima, T., and Yamamoto, M. 2006. The Caenorhabditis elegans homologue of deleted in azoospermia is involved in the sperm/oocyte switch. Mol. Biol. Cell 17: 3147-3155.

Piccioni, F., Zappavigna, V., and Verrotti, A.C. 2005. Translational regulation during oogenesis and early development: the cappoly(A) tail relationship. C. R. Biol. 328: 863-881.

Reijo, R., Lee, T.Y., Salo, P., Alagappan, R., Brown, L.G., Rosenberg, M., Rozen, S., Jaffe, T., Straus, D., Hovatta, O., et al. 1995. Diverse spermatogenic defects in humans caused by $\mathrm{Y}$ chromosome deletions encompassing a novel RNA-binding protein gene. Nat. Genet. 10: 383-393.

Reynolds, N., Collier, B., Maratou, K., Bingham, V., Speed, R.M., Taggart, M., Semple, C.A., Gray, N.K., and Cooke, H.J. 2005. Dazl binds in vivo to specific transcripts and can regulate the premeiotic translation of Mvh in germ cells. Hum. Mol. Genet. 14: 3899-3909.

Ruggiu, M. and Cooke, H.J. 2000. In vivo and in vitro analysis of homodimerization activity of the mouse Dazl1 protein. Gene 252: $119-126$.
Ruggiu, M., Speed, R., Taggart, M., McKay, S.J., Kilanowski, F., Saunders, P., Dorin, J., and Cooke, H.J. 1997. The mouse Dazla gene encodes a cytoplasmic protein essential for gametogenesis. Nature 389: 73-77.

Saunders, P.T.K., Turner, J.M.A., Ruggiu, M., Taggart, M., Burgoyne, P.S., Elliott, D., and Cooke, H.J. 2003. Absence of mDazl produces a final block on germ cell development at meiosis. Reproduction 126: 589-597.

Schrans-Stassen, B.H., Saunders, P.T., Cooke, H.J., and de Rooij, D.G. 2001. Nature of the spermatogenic arrest in Dazl -/- mice. Biol. Reprod. 65: 771-776.

Sun, F., Greene, C., Turek, P.J., Ko, E., Rademaker, A., and Martin, R.H. 2005. Immunofluorescent synaptonemal complex analysis in azoospermic men. Cytogenet. Genome Res. 111: 366-370.

Tay, J. and Richter, J.D. 2001. Germ cell differentiation and synaptonemal complex formation are disrupted in CPEB knockout mice. Dev. Cell 1: 201-213.

Teng, Y.N., Lin, Y.M., Lin, Y.H., Tsao, S.Y., Hsu, C.C., Lin, S.J., Tsai, W.C., and Kuo, P.L. 2002. Association of a single-nucleotide polymorphism of the deleted-in-azoospermia-like gene with susceptibility to spermatogenic failure. J. Clin. Endocrinol. Metab. 87: 5258-5264.

Topping, D., Brown, P., Judis, L., Schwartz, S., Seftel, A., Thomas, A., and Hassold, T. 2006. Synaptic defects at meiosis I and nonobstructive azoospermia. Hum. Reprod. 21: 3171-3177.

Tsui, S., Dai, T., Roettger, S., Schempp, W., Salido, E.C., and Yen, P.H. 2000a. Identification of two novel proteins that interact with germ-cell-specific RNA-binding proteins DAZ and DAZL1. Genomics 65: 266-273.

Tsui, S., Dai, T., Warren, S.T., Salido, E.C., and Yen, P.H. $2000 \mathrm{~b}$. Association of the mouse infertility factor DAZL1 with actively translating polyribosomes. Biol. Reprod. 62: 1655-1660.

Tung, J.Y., Rosen, M.P., Nelson, L.M., Turek, P.J., Witte, J.S., Cramer, D.W., Cedars, M.I., and Pera, R.A. 2006a. Variants in deleted in azoospermia-like (DAZL) are correlated with reproductive parameters in men and women. Hum. Genet. 118: 730-740.

Tung, J.Y., Rosen, M.P., Nelson, L.M., Turek, P.J., Witte, J.S., Cramer, D.W., Cedars, M.I., and Reijo-Pera, R.A. 2006b. Novel missense mutations of the deleted-in-azoospermia-like (DAZL) gene in infertile women and men. Reprod. Biol. Endocrinol. 4: 40.

Urano, J., Fox, M.S., and Reijo Pera, R.A. 2005. Interaction of the conserved meiotic regulators, BOULE (BOL) and PUMILIO-2 (PUM2). Mol. Reprod. Dev. 71: 290-298.

Venables, J.P., Ruggiu, M., and Cooke, H.J. 2001. The RNA-binding specificity of the mouse Dazl protein. Nucleic Acids Res. 29: 2479-2483.

Xu, E.Y., Moore, F.L., and Pera, R.A. 2001. A gene family required for human germ cell development evolved from an ancient meiotic gene conserved in metazoans. Proc. Natl. Acad. Sci. 98: 7414-7419.

Yuan, L., Liu, J.G., Hoja, M.R., Wilbertz, J., Nordqvist, K., and Hoog, C. 2002. Female germ cell aneuploidy and embryo death in mice lacking the meiosis-specific protein SCP3. Science 296: 1115-1118.

Yuan, L., Liu, J.G., Zhao, J., Brundell, E., Daneholt, B., and Hoog, C. 2000. The murine SCP3 gene is required for synaptonemal complex assembly, chromosome synapsis, and male fertility. Mol. Cell 5: 73-83. 

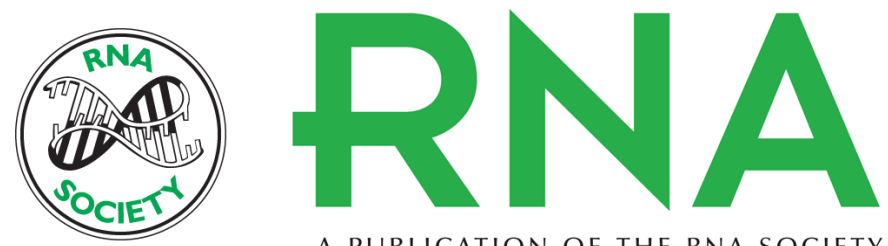

A PUBLICATION OF THE RNA SOCIETY

\section{Translation of the synaptonemal complex component Sycp3 is enhanced in vivo by the germ cell specific regulator Dazl}

Nicola Reynolds, Brian Collier, Victoria Bingham, et al.

RNA 2007 13: 974-981 originally published online May 25, 2007

Access the most recent version at doi:10.1261/rna.465507

\section{References This article cites 53 articles, 14 of which can be accessed free at: http://rnajournal.cshlp.org/content/13/7/974.full.html\#ref-list-1}

\section{License} Email Alerting
Service 